\title{
CAPTURA, TRATAMIENTO Y REUTILIZACIÓN DE AGUA GRIS Y DE LLUVIA EN RESIDENCIA UNIFAMILIAR
}

\section{ARTÍCULO DE REVISIÓN}

MONTEIRO, Otávio Pinetti ${ }^{1}$

LIMA, Gemael Barbosa ${ }^{2}$

MONTEIRO, Otávio Pinetti. LIMA, Gemael Barbosa. Captura, tratamiento y reutilización de agua gris y de lluvia en residencia unifamiliar. Revista Científica Multidisciplinar Núcleo do Conhecimento. Año 05, Ed. 01, Vol. 01, págs. 98-113. Enero de 2020. ISSN: 2448-0959, Enlace de acceso: https://www.nucleodoconhecimento.com.br/medio-ambiente/agua-de-ceniza

\section{RESUMEN}

El objetivo de esta obra es presentar una revisión bibliográfica, cuyo tema es la reutilización del agua por la lluvia, así como la reutilización de aguas grises en una residencia unifamiliar. Para ello, la metodología constituye una búsqueda de información en libros, revistas, artículos, tesis de maestría y tesis doctorales. Reutilizar conscientemente, formas de capturar agua de una residencia, ya sea a partir de la lluvia que cae sobre las canaletas o utilizada en los métodos de captura en los fregaderos de la cocina y / o tanques de áreas de servicio que recibirán el tratamiento necesario y serán reutilizados. Estos tratamientos actúan de manera eficiente y proporcionan el uso de esta agua para fines menos nobles que hace que el consumo

\footnotetext{
${ }_{1}^{1}$ Postgrado en Ingeniería Ambiental y Seguridad Ocupacional (Faveni), Postgrado en Sostenibilidad en el Medio Ambiente Construido (Ifes), Postgrado en Enseñanza de La Educación Superior (Faveni), Graduado en Ingeniería Civil (Unesc), Graduado en Matemáticas.

${ }^{2}$ Máster en Ingeniería Ambiental, profesor en la UNESC.
} 
sea más racional y económico, cooperando para el mantenimiento de los recursos ambientales.

Palabras clave: Agua, reutilización, captura, tratamiento, residencia.

\section{INTRODUCCIÓN}

Como afirma Zampieron (2005), el agua se ha convertido en un símbolo común de la humanidad, valorada en todas las religiones y culturas, también un símbolo de igualdad social, porque la crisis del agua es, sobre todo, la distribución, los recursos y no sólo en su absoluta escasez . Con el claro objetivo de reducir el consumo de agua, a través de las nuevas tecnologías, comenzó el desarrollo de varios programas de uso racional.

Estos problemas asociados corroboran la discusión de la viabilidad de hacer el uso de agua de lluvia y aguas grises para actividades fundamentales en una residencia. Esta posibilidad de reutilización sin duda dará lugar a la disminución del consumo de agua tratada que proporcionan las empresas de saneamiento, además, se reducirá la demanda de costes con el uso de agua potable y la reducción de los riesgos derivados de inundaciones en períodos de lluvias intensas.

Por lo tanto, la investigación abordada tiene como objetivo demostrar cómo se realiza la reutilización del agua de la lluvia junto con la reutilización de agua gris en una residencia unifamiliar, a partir de la presentación de un estudio de caso. Toda la base del trabajo se encontró a través de la información en libros, revistas, artículos y tesis de maestría, en las que los autores, así como los investigadores de la zona, laboratorio e investigación académica se utilizaron para apoyar sus resultados.

Aunque el agua es un recurso renovable, su oferta no satisface la demanda que con el tiempo ha ido aumentando sustancialmente. Con esto siempre habrá un déficit, que se acentúa cada vez más por el uso irracional y diversos residuos. Por lo tanto, es cada vez más necesario establecer una nueva relación entre el hombre y el agua, ya que esto se están ampliando las opciones de uso racional y añadiendo a este factor, 
alternativas para aprovechar eficientemente, ya sea a través de la captura y el tratamiento de como se aborda en el trabajo o por métodos más específicos que aún no son bien recibidos por la población por tener el precio de inversión muy alto.

\section{MARCO TEÓRICO}

[...] En combinación con la capacidad de disolución antes mencionada, el agua actúa como un medio de transporte - en escorrente superficial y subterránea - permitiendo que las características del mismo curso de agua cambien temporal y espacialmente. (LEBANON, 2010, p.15)

La potabilización de las aguas naturales con fines de consumo humano tiene como función esencial adaptar el agua cruda a los límites físicos, químicos, biológicos y radiactivos establecidos por la Ordenanza 2914, haciendo que el efluente de la estación no pueda transmitir perjudiciales para la población suministrada.

[...] El tratamiento del agua consiste en la eliminación de partículas suspendidas y coloidales, materia orgánica, microorganismos y otras sustancias nocivas para la salud humana, tal vez presentes en aguas naturales, a los costos más bajos de implantación, operación y mantenimiento, y generando el menor impacto ambiental en las áreas circundantes. (LIBÂNIO, 2010, p.135).

\subsection{USO DEL AGUA EN EL CONTEXTO DE LA CONSTRUCCION CIVIL}

Según Mayok (2009), existen otras normas, como NBR 7229/1993, que tiene el tamaño del sistema de agua servido; y las directrices para la conservación y reutilización del agua en los edificios preparados por la Unión de la Construcción Civil (Sinduscon), que presenta varias formas de reutilizar el agua, incluso en el lavado de áridos para su uso en la construcción civil.

Según Sickermann (2005) citado por la PROSAB, 2006 como un recurso extremadamente importante para la construcción, el agua se utiliza en todas las 
etapas de una obra, desde la producción de hormigón incluso para satisfacer las necesidades básicas de los trabajadores, y su escasez ya se siente en obras de construcción en diferentes lugares del país.

Según el vicepresidente de la Unión de la Construcción (Sinduscon-MG)Geraldo Jardim Linhares Júnio, aunque la situación aún no es grave, las empresas de construcción han optado por sustituir por métodos convencionales por métodos de construcción en seco, con el fin de crear cierta independencia del agua (MARIANE, 2014).

\subsection{USOS MÚLTIPLES DEL AGUA}

Basado en la investigación de Tomaz (2005) con respecto a la reutilización del agua de lluvia, el uso de medidas y técnicas es excelente para evitar y remediar problemas sobre la falta de agua potable, incluso sin leyes que regulen su uso. El uso de métodos apropiados y el uso alterno con agua de las alcantarillas domésticas sería una manera inteligente de conservar el agua para fines nobles.

Como indica Caubet (2006), representante de organizaciones no gubernamentales (ONG) en el Consejo Nacional de Recursos Hídricos (CNRH), incluso si el Código del Agua (Decreto Federal No. 24.643, a partir del 10/07/1934) presenta la prioridad absoluta del uso del agua para las necesidades básicas de los seres humanos (desicción y usos domésticos), la mayor retirada tiene lugar en el ámbito de la agricultura (62,7\%), luego se produce a través del consumo humano (17,9\%) y uso industrial (14\%) y, por último, en el consumo animal $(5,4 \%)$, como se muestra en la Figura 01. 
Figura 01-Consumo de agua por actividad en Brasil.

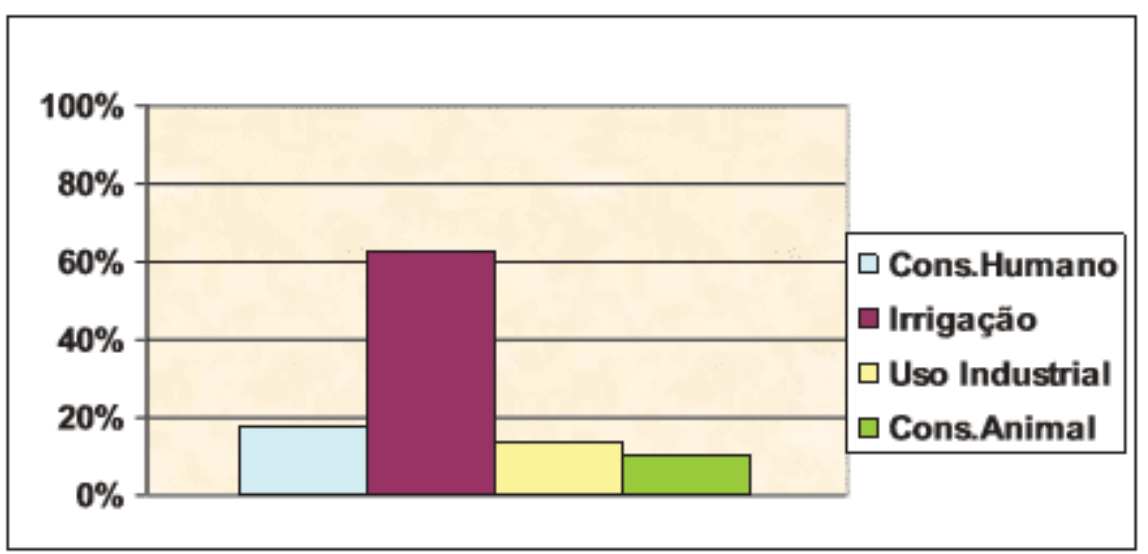

Fuente: ANA (2002) citada por MANUAL FIESP/CIESP (2006).

\subsubsection{USOS DE AGUA NO POTABLE}

Estudios realizados en Brasil y en el extranjero muestran que en una residencia, gran parte del consumo de agua se centra en los baños, la descarga de baños, fregadero de cocina y ropa de lavado. En promedio, el $40 \%$ del agua total consumida en una residencia se utiliza de forma no potable. (GONÇALVES, 2009).

La necesidad de analizar la cantidad de agua distribuida para satisfacer las actividades básicas de consumo e higiene es motivo de discusión entre varios autores, ya que podemos analizar en la Figura 02. Una definición propuesta por Peter Gleick, que figura en el Requisito Básico de Agua (BWR), es que $50 \mathrm{~L} / \mathrm{hab} /$ día es una cantidad suficiente para satisfacer estas necesidades (BIO, 2002). 
Figura 02 - Distribución del consumo de agua en los hogares por cada aparato

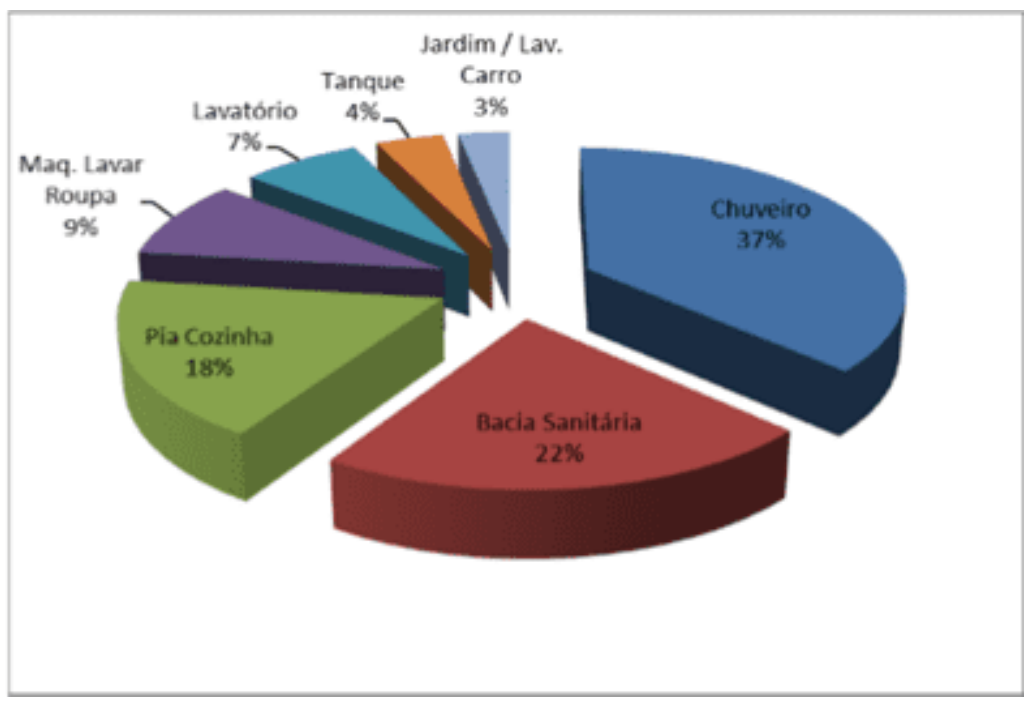

Fuente: Adaptado de Martins y Memelli (2011)

\subsubsection{USOS DE AGUA POTABLE}

El agua potable es todo el agua adecuada para el consumo. Siendo un líquido incoloro, inodoro, insípido e deshuesado, esencial para la supervivencia humana, como afirma Hespanhol (2002).

\subsubsection{TRATAMIENTO}

No es esencial que el agua en primavera presente todos los indicadores adecuados para el consumo, ya que según Braga (2005) hay formas de cambiar sus características para que sea compatible con las necesidades de salud pública.

También según Braga (2005), los principales métodos de tratamiento del agua son: decantación, floculación, filtración, desinfección, eliminación de dureza, aireación, eliminación de hierro y manganeso, eliminación del sabor y olor, control de la corrosión y fluoración, que son siempre interconectados y que la falta de un tratamiento adecuado de algunos de estos elementos puede alterar la calidad final del agua. 


\section{MATERIALES Y MÉTODOS}

La metodología de este artículo consistió en investigación bibliográfica en libros, revistas científicas, tesis doctorales y tesis de maestría. La revisión se limitó a formas de capturar y aprovechar el agua de lluvia, además, también se discutió sobre el uso de aguas grises. Este trabajo se centró en tratar las técnicas antes mencionadas en el contexto de la residencia unifamiliar.

Para basar mejor la revisión bibliográfica propuesta en este artículo, se presentó un estudio de caso de Oliveira (2005) en el municipio de Palhoa, situado a $15 \mathrm{~km}$ de Florianópolis, en el estado de Santa Catarina. Después de presentar los resultados de la investigación de Oliveira (2005), la discusión se llevó a cabo trayendo otros estudios similares.

En las siguientes secciones se presentará el área de estudio y el objetivo, así como las características de la residencia, entre otros aspectos del caso de estudio elegido para citar este artículo.

\section{1 ÁREA DE ESTUDIO}

El municipio elegido para ejecutar el proyecto fue Palhoa, situado a $15 \mathrm{~km}$ de Florianópolis entre la costa y la Serra do Mar, en meridiano 48040'04" longitud oeste y paralelo 280 38' 43" latitud sur. Tiene una superficie de $323 \mathrm{~km} 2$, de los cuales el $73,0 \%$ del territorio se considera zona de conservación permanente. La población del municipio, según IBGE (2004), es de 113.312 habitantes, según (PALHO-A, 2004). EI Palhoa, según Setti (1994), está a tres metros sobre el nivel del mar, en una llanura costera con manglares, restingas y macizos rocosos de la Serra do Mar. El clima, según el autor, es húmedo con una temperatura media de 25oC. La precipitación anual de 1969 a 2002, según CLIMERH/EPAGRI, es de 1706 mm. 


\subsection{OBJETO DE ESTUDIO}

El estudio se basó en dos residencias distintas y se encuentra a una distancia de 2 km entre sí. Residencia 01 tiene una superficie de 131,36m2 y la residencia 02 tiene una superficie de 143,27 m2. En la residencia 01, los puntos de consumo se encuentran en el baño, la zona de servicio y la cocina. El baño consta de ducha, lavamanos y aseo con descarga de válvulas. En la cocina, hay un toque en el fregadero. En el área de servicio, hay una lavadora que utiliza 100 litros de agua por ciclo y un grifo ubicado en el tanque. En la residencia 02, los puntos de consumo de agua están situados en tres baños, cocina y área de servicio. En los tres baños hay dos duchas, tres grifos para lavabo, dos con mezcladores y tres descargas sanitarias. En la cocina, hay un grifo con mezclador y en la zona de servicio hay una lavadora con un consumo de 801 por ciclo y un grifo en el tanque. En ambas residencias prácticamente no se utiliza agua potable para lavar aceras, coches o riego de jardín. El uso del agua utilizada por la lavadora ya ocurre en ambas residencias, almacenándola en tanques para su posterior reutilización en aceras y jardines.

\subsection{SISTEMA DE RECOGIDA Y ALMACENAMIENTO DE AGUA DE AGUA Y AGUA DE LLUVIA}

\subsubsection{DETERMINACIÓN DE LAS ÁREAS DE COBERTURA}

Para almacenar el agua procedente de la lluvia es necesario inspeccionar las zonas de cubierta de ambas residencias. La determinación de estas áreas se hizo a través de un vasto análisis de los planes de la cobertura de las residencias propuestas siendo el mismo importante en la captura.

\subsubsection{COLECCIÓN DE AGUA DE TECHO}

El agua de lluvia es capturada por canalones y llevada al depósito inferior a través de conductores verticales, escalada según NBR 10844 (ABNT, 1989). Esta norma se ocupa de las instalaciones de construcción de agua de lluvia. 


\subsubsection{EMBALSE PARA LA CAPTURA DE AGUA DE LLUVIA}

Para alcanzar el volumen del embalse que almacena el agua de la lluvia, se analizaron las áreas de contribución de la cobertura de viviendas, el consumo diario de agua por habitante, en la precipitación de la región y en el coeficiente de uso del agua. Lluvia. Este coeficiente muestra el porcentaje que se almacena, ya que el resto se utiliza para limpiar el techo, canalones y tuberías y evaporación. Para este estudio, se adoptará que se recogerá el $80 \%$ del agua que cae sobre el techo.

En cada residencia habrá dos embalses, uno más bajo que hará el almacenamiento de agua de lluvia recogida y el superior que se utilizará para distribuir las aguas para el consumo. El fondo será de fibra de vidrio enterrado en el terreno. La eliminación del primer agua de lluvia se llevará a cabo a lo largo de la tubería situada antes de que el agua entre en el depósito. La primera lluvia se mantiene en este tubo, evitando que la mayoría de las impurezas lleguen al depósito. Para la retención de residuos más grandes se instalará en la salida de las canaletas una rejilla de pvc flexible. Al final de la tubería de entrada de agua en el depósito, se instalará una reducción y una rodilla de 900 con un diámetro mayor que el tubo de entrada. El aumento de la superficie disminuye el impacto del agua de lluvia recogida en el fondo del depósito, evitando así la agitación de partículas depositadas (Figura 03).

Figura 03 - Modelo de embalse utilizado en el sistema de captura y almacenamiento.

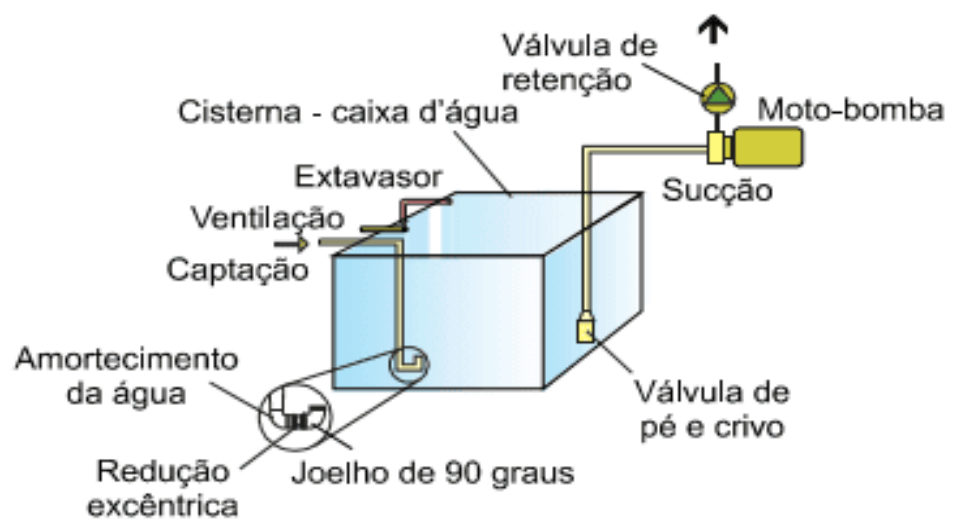

Fuente: ANA (2002) citada por MANUAL FIESP/CIESP (2006). 
El depósito superior se alimentará a través de un sistema de bomba de motor. Entre el depósito superior de agua de lluvia y el depósito de agua potable debe haber un sistema "by pass" que abastecerá de agua potable a la falta de agua de lluvia.

\subsubsection{RESERVORIO PARA LA REUTILIZACIÓN DEL AGUA}

Los embalses de reutilización tendrán volúmenes relacionados con el volumen de aguas residuales secundarias (agua de baño, lavabo, lavadora y agua arasda) generados en ambas residencias. Estos volúmenes se verificarán a través de una encuesta realizada en el lugar del consumo de agua. En cada residencia habrá dos embalses: un nivel inferior implantado para el almacenamiento de agua, y un superior sólo para la distribución del consumo. El depósito inferior será de fibra de vidrio y enterrado en el suelo. Antes de llegar al embalse, las aguas residuales secundarias se someterán a una caja de eliminación de arena para la retención de sus residuos, y a un sistema de tratamiento vegetal llamado zona radicular. Después de almacenar el agua ya con el tratamiento adecuado, se llevará al depósito superior a través de un sistema de bomba de motocicleta y luego alimentar los puntos de uso.

\section{RESULTADOS}

Para el proyecto presentado por Oliveira (2005) en el que se realiza la captura, almacenamiento y distribución de agua de lluvia y reutilización, se realizó un estudio de los usos finales de esta agua en el municipio de Palhoa. A partir de la determinación de estos usos finales, se puede determinar el volumen de agua necesario para el uso del agua de lluvia y el volumen de aguas residuales secundarias disponibles para su reutilización. Después de determinar estos volúmenes, los sistemas se escalan para el agua de lluvia y para la reutilización de aguas grises, y luego se realiza el análisis económico para determinar la viabilidad de los sistemas implantados. 


\subsection{PUNTOS DE MAYOR CONSUMO EN RESIDENCIA 01}

Tras la corrección realizada a través de los métodos de sensibilidad, el lugar donde el mayor consumo de agua fue la ducha con el $32,8 \%$ del consumo total de la residencia, seguido del aseo con el $30,4 \%$ y el grifo de cocina con $28,0 \%$, según la Tabla 01.

Cuadro 01 - Descripción del consumo de agua en los puntos de residencia 01

\begin{tabular}{lccccc}
\hline \multirow{2}{*}{ Aparelho } & \multicolumn{4}{c}{ Consumo (litros) } & \multirow{2}{*}{ \% } \\
\cline { 2 - 5 } & $\mathbf{H}_{\mathbf{1}}$ & $\mathbf{M}$ & $\mathbf{H}_{2}$ & Total & \\
\hline Chuveiro & 1528,9 & 1662,6 & 2092,8 & 5284,3 & 32,8 \\
Lavatório & 92,8 & 132,1 & 73,8 & 298,7 & 1,9 \\
Vaso Sanitário & 1465,1 & 2289,6 & 1138,6 & 4893,2 & 30,4 \\
Barba & 75,6 & - & 55,2 & 130,8 & 0,8 \\
Máquina Lavar Roupa & 333,3 & 333,3 & 333,3 & 1000,0 & 6,2 \\
Torneira Cozinha & 1505,0 & 1505,0 & 1505,0 & 4515,0 & 28,0 \\
\hline TOTAL & & & & $\mathbf{1 6 1 2 2 , 0}$ & $\mathbf{1 0 0 , 0}$ \\
\hline
\end{tabular}

Fuente: Oliveira, 2005

\subsection{PUNTOS DE MAYOR CONSUMO EN RESIDENCIA 02}

En la residencia 02, tras la corrección, también se observó el mayor consumo en la ducha, con el 45,6\% del consumo total de la residencia, seguido del aseo con el 25,6\% y el fregadero de la cocina con el $13,5 \%$, como se presenta en la Tabla 02.

Cuadro 02 - Descripción del consumo de agua en los puntos de residencia 02

\begin{tabular}{lcccc}
\hline \multirow{2}{*}{ Aparelho } & \multicolumn{3}{c}{ Consumo (litros) } & \\
\cline { 2 - 4 } & $\mathbf{M}$ & $\mathbf{H}$ & Total & $\%$ \\
\hline Chuveiro & 1339,2 & 1762,8 & 3102,0 & 45,6 \\
Lavatório & 112,0 & 66,2 & 178,3 & 2,6 \\
Vaso Sanitário & 986,7 & 755,5 & 1742,2 & 25,6 \\
Barba & - & 300,0 & 300,0 & 4,4 \\
Máquina Lavar Roupa & 280,0 & 280,0 & 560,0 & 8,2 \\
Torneira Cozinha & 460,8 & 460,8 & 921,6 & 13,5 \\
\hline TOTAL & & & $\mathbf{6 8 0 4 , 0}$ & $\mathbf{1 0 0 , 0}$ \\
\hline
\end{tabular}

Fuente: Oliveira, 2005 


\subsection{UTILIZACIONES FINALES PARA AGUA Y AGUA RE-SO}

Para determinar el volumen diario disponible para la reutilización del agua y el volumen diario necesario para el uso del agua de lluvia se obtiene a través de los usos finales del agua después de la corrección realizada en el consumo estimado. Las tablas 03 y 04 observan la cantidad de agua utilizada para la reutilización, así como la cantidad necesaria para el suministro mediante reutilización y el volumen necesario para el uso de agua de lluvia. La cantidad necesaria de agua disponible para utilizar como reutilización se obtuvo del consumo en la ducha, lavabo, agua utilizada para afeitar y en la lavadora, totalizando, para la residencia 01, aproximadamente 240 litros, lo que equivale al $41,9 \%$ del consumo residencia total. Con el agua de reutilización sólo se puede abastecer el aseo, con una necesidad diaria de 175,0 litros, alrededor del 30\% del consumo de la residencia. Por lo tanto, hay suficiente agua disponible para abastecer el inodoro. Usando agua de lluvia, puede suministrar el inodoro y la lavadora. En la residencia 01, hay una necesidad diaria de 210,5 litros, alrededor del $36,6 \%$ del consumo total de la residencia.

Cuadro 03 - Consumo para reutilización y uso de agua de lluvia en la residencia 01.

\begin{tabular}{|c|c|c|c|c|c|c|c|c|c|}
\hline \multirow{3}{*}{ Aparelhos } & \multicolumn{9}{|c|}{ Consumo } \\
\hline & \multicolumn{3}{|c|}{ Reúso - Disponível } & \multicolumn{3}{|c|}{ Reúso - Necessário } & \multicolumn{3}{|c|}{ Água Chuva } \\
\hline & $\begin{array}{l}\text { Diário } \\
\text { (litros) }\end{array}$ & $\begin{array}{l}\begin{array}{l}\text { Mensal } \\
\text { (litros) }\end{array} \\
\end{array}$ & $\%$ & $\begin{array}{l}\text { Diário } \\
\text { (litros) }\end{array}$ & $\begin{array}{c}\text { Mensal } \\
\text { (litros) }\end{array}$ & $\%$ & $\begin{array}{l}\text { Diário } \\
\text { (litros) }\end{array}$ & $\begin{array}{c}\text { Mensal } \\
\text { (litros) }\end{array}$ & $\%$ \\
\hline Chuveiro & 188,7 & 5284,3 & 32,8 & - & - & - & - & - & - \\
\hline Lavatório & 10,7 & 298,7 & 1,9 & - & - & - & - & - & - \\
\hline Vaso Sanitário & - & - & - & 174,8 & 4893,3 & 30,4 & 174,8 & 4893,3 & 30,4 \\
\hline Barba & 4,7 & 130,8 & 0,8 & - & - & - & - & - & - \\
\hline Máquina Lavar $\mathrm{F}$ & 35,7 & 1000,0 & 6,2 & - & - & - & 35,7 & 1000,0 & 6,2 \\
\hline TOTAL & 239,8 & 6713,8 & 41,9 & 174,8 & 4893,3 & 30,4 & 210,5 & 5893,3 & 36,6 \\
\hline
\end{tabular}

Fuente: Oliveira, 2005

Cuadro 04 - Consumo para reutilización y uso de agua de lluvia en la residencia 02.

Fuente: Oliveira, 2005 


\subsection{AHORROS FINALES CON LA CAPTURA, ALMACENAMIENTO Y TRATAMIENTO DEL AGUA DE LLUVIA Y RESO}

El Cuadro 05 muestra los ahorros con agua potable, refiriéndose al uso del sistema de uso del agua a partir de la lluvia, la implementación del sistema de agua de reutilización y la implementación del agua de lluvia y la reutilización conjunta, en los hogares 01 y 02 , en moneda ( $y$ en porcentaje.

En la residencia 01 , hay un ahorro anual con agua potable del $35,5 \%$ debido a la implementación del sistema de uso de agua de lluvia, y alrededor del $30,4 \%$ con el uso del sistema de reutilización. La implementación del conjunto de agua de lluvia y reutilización produjo ahorros con agua potable del $36,4 \%$.

Para la residencia 02, implementando un sistema de uso de agua de lluvia, se obtuvo una economía con agua potable del $33,6 \%$ y con la implementación de la reutilización, $25,6 \%$. La economía total con ambas formas de captura fue del $33,8 \%$.

Cuadro 05 - Ahorro anual en residencias 1 y 2.

\begin{tabular}{lcccc}
\hline \multirow{2}{*}{\multicolumn{1}{c}{ Economia }} & \multicolumn{4}{c}{ Economia } \\
\cline { 2 - 5 } & \multicolumn{2}{c}{ Residência 1 } & \multicolumn{2}{c}{ Residência 2 } \\
\cline { 2 - 5 } & $\mathbf{R}$ & $\mathbf{\%}$ & $\mathbf{R}$ & $\mathbf{6}$ \\
\hline Economia anual - água de chuva & 117,10 & 35,5 & 46,75 & 33,6 \\
Economia anual - reúso de água & 100,05 & 30,4 & 35,60 & 25,6 \\
Economia anual - água de chuva + reúso & 119,84 & 36,4 & 47,01 & 33,8 \\
\hline
\end{tabular}

Fuente: Oliveira, 2005

\section{DISCUSIÓN}

Para el proyecto presentado en la ciudad de Palhoa abordado en este artículo, el sistema utilizó componentes simples que satisfacían todas las necesidades básicas y que además de ser un método fácil, puede generar ahorros para los residentes donde la inversión inicial fue baja y la regreso sucedió en poco tiempo. La investigación también compara el mismo método aplicado en dos unidades diferentes, que tiene 
como objetivo establecer los métodos de construcción y su contribución a la recolección y almacenamiento de agua para su reutilización.

Otros autores establecen diferentes sistemas para la captura y tratamiento del agua gris y de lluvia, donde la economía varía según las inversiones establecidas por cada modelo y también de acuerdo con la eficiencia del sistema.

Según el trabajo de Silveira (2008), la "Línea Europa", basada en un estudio alemán de tratamiento de aguas pluvatenes, comprende sistemas que suministrarán uso doméstico y externo, sirviendo también a áreas de mayor captura. Es ideal para residencias que aún están en fase de construcción, ya que permite una mayor integración entre los sistemas de agua potable y de agua de lluvia. Este método según el investigador utilizará una bomba, además de otros accesorios como el freno de agua (para reducir el torbellino en la cisterna), filtro flotante para permitir una mayor calidad del agua capturada y multisifón para no dejar que los insectos entrar en el sistema. El dimensionamiento del embalse para su almacenamiento será el mismo que el cubierto por los trabajos realizados en la ciudad de Palhoa, donde se define a través de la previsión de consumo, la base de captura y el tiempo de secado para el sitio. También puede conectar el sistema de aguas pluvatas con el agua de la red pública de abastecimiento para que en los momentos de falta de agua la integración entre los dos sistemas garantice la calidad y el suministro, según la Figura 04. 
Figura 04: Modelo de la línea Europa abordada por la mestranda Bruna Quick da Silveira

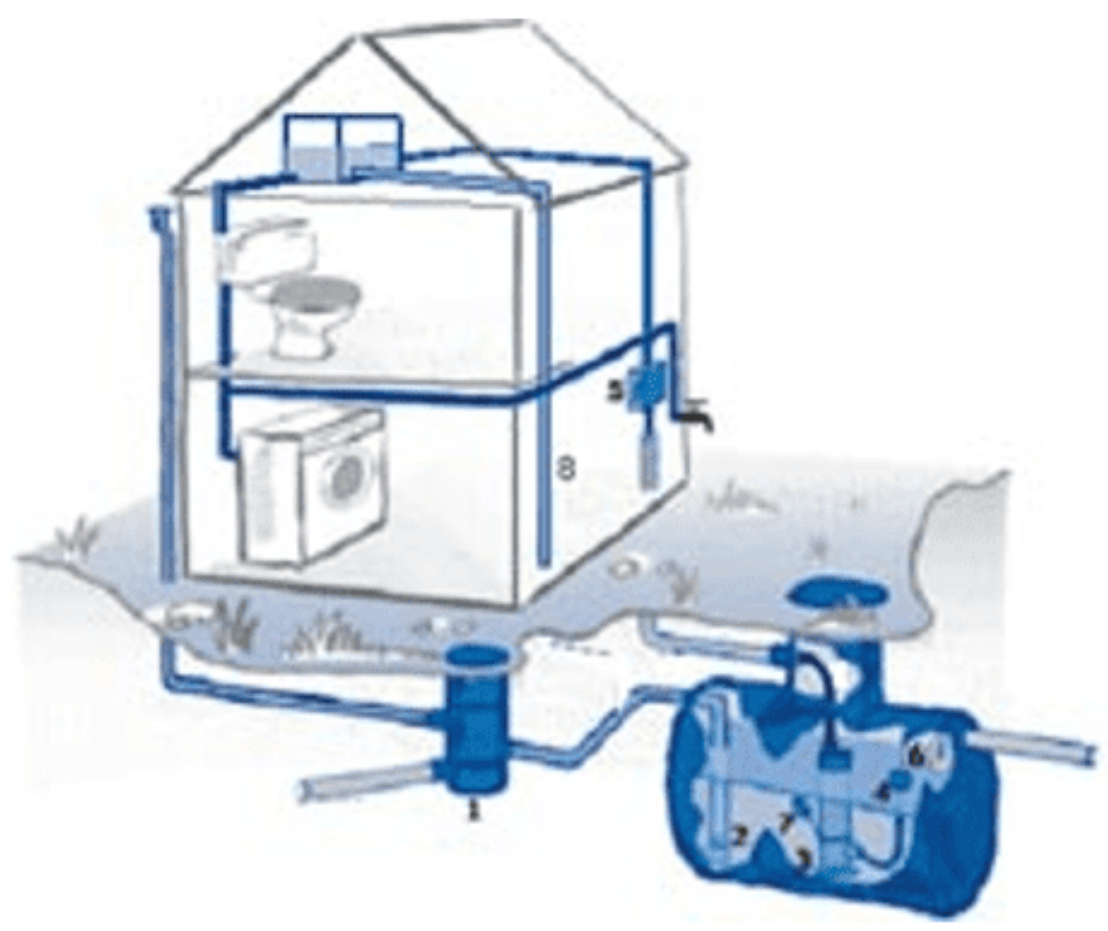

1.Filtro tipo vortex

2. Freio d'água

3. Bomba submersivel

4. Filtro flutuante

5. Central de controle/ interligação com rede pública

6. Multisifäo

7. Bóia de nível

8. Alimentação dos Pontos de Consumo a partir da Caixa d'água superior

Fuente: AQUASTOCK, 2008.

Los dos sistemas enfrentados son eficientes, pero utilizan diferentes formas de capturar, tratar y distribuir el agua recolectada, donde cada método tiene su particularidad. La eficiencia de ambos es satisfactoria, lo que hace que los dos esquemas sean habituales y económicos, teniendo como factor determinante la elección de la inversión inicial más adecuada que cada uno demandará, siendo la utilizada en la ciudad de Palhoa el método más sencillo, que termina una inversión inicial menor que la presentada por la mestranda, denominada sistema de "Línea Europa". 


\section{CONCLUSIÓN}

A partir del objetivo de este artículo, se puede percibir que el interés de la sociedad es pequeño cuando el tema de la agenda es la instalación de un sistema capaz de reutilizar el agua que antes se descartaba. Esto se debe a que existe un cierto perjuicio con la reutilización del agua de los efluentes domésticos y también debido al mayor riesgo de contaminación asociado con el sistema de mantenimiento que puede promover daños a la salud de los usuarios directos o indirectos del sistema. Un método de reutilización adecuado y operado conscientemente aumenta la eficiencia y reduce cualquier tipo de contaminación que pueda ocurrir por parte del operador o del sistema en su conjunto.

El modelo de captura presentado en esta obra o cualquier otro disponible en el mundo requiere un coste de instalación inicial muy alto, pero a largo plazo debe tener ahorros en el volumen de agua utilizada en la residencia, así como en la calidad del agua que se recibe , porque al tratar y reutilizar el agua que una vez volvió a la naturaleza habrá la preservación de la calidad de este bien que, además de finito, es esencial para el bienestar y para la calidad de la vida humana.

\section{REFERENCIAS}

ABNT - Associação Brasileira de Normas Técnicas. NBR 10844 - Instalações Prediais de Águas Pluviais. Rio de Janeiro (1989).

ABNT Associação Brasileira de Normas Técnicas. NBR 5626 - Instalações Prediais de Água Fria. Rio de Janeiro (1998).

ANA - Agência Nacional de Águas. A Evolução da Gestão dos Recursos Hídricos no Brasil / The Evolution of Water Resources Management in Brazil. Brasília; ANA, 2002

AQUASTOCK - Água da Chuva. Sistema de Reaproveitamento da Água da Chuva. Disponível em: <http://www.engeplasonline.com.br> Acesso em: 21/08/2008 
BIO: Revista Brasileira de Saneamento e Meio Ambiente. Água: o ouro azul do século XXI. Rio de Janeiro, RJ, v.11, n. 21, jan./mar. 2002

BIO: Revista Brasileira de Saneamento e Meio ambiente. Centronetwork. Rio de Janeiro, $n^{\circ}$ 21. Ano XI. 2002. p.50

BRAGA, BENEDITO et al. Introdução à Engenharia Ambiental O desafio do desenvolvimento sustentável. 2ª edição. São Paulo: Pearson Prentice Hall, 2005.

CAUBET, Christian Guy. A água doce nas relações internacionais, ano 2006.

FIORI, Simone. Avaliação Qualitativa E Quantitativa Do Potencial De Reuso De Água Cinza Em Edifícios Residenciais Multifamiliares. Dissertação de Mestrado da Universidade de Passo Fundo (RS), 2005. Disponível: <http//www.usp.br>. Acesso em 12/11/2016.

GONÇALVES, R. F. (Coord.). Conservação de água e energia em sistemas prediais e públicos de abastecimento de água. Rio de Janeiro: ABES, 2009

HESPANHOL, I. Potencial de Reúso de Água no Brasil: Agricultura, Indústria, Municípios, Recarga de Aquíferos. Recursos Hídricos: APRH, Portugal, v. 23, n.2, p.43-65, nov. 2002.

LIBÂNIO, M. Fundamentos de qualidade e tratamento de água, 3eiç̧ão Campinas: Átomo, 2010.

MARIANE, Aline -Construtoras adotam sistemas de reuso e aproveitamento de água de chuva, além de métodos de construção seca, para contornar falta de água nas obras. Disponível em: http://construcaomercado.pini.com.br/negocios-incorporacaoconstrucao/161/artigo333851-1.aspx >Dez,2014. Acesso em: 12 Dez.2016.

MAY, Simone. Conservação e Reúso de Água em Edifícios: Reúso De Águas Cinzas e Aproveitamento De Águas Pluviais Para Consumo Não Potável. Escola Politécnica da Universidade de São Paulo (USP),2009. 
MAYOK, SIMONE. Caracterização, tratamento e reuso de águas cinzas e aproveitamento de águas pluviais em edificações. São Paulo, junho, 2009.

MIERZWA, J.C; VERAS,L.R; SILVA,M.C. Avaliação do Desempenho de Membrana de Ultrafiltração para Tratamento de Água Potável. VIII Seminário IberoAmericano. São Paulo: 2005.

OLIVEIRA, N. M.; SILVA, M. P. da.; CARNEIRO, V. A. Reúso da água: um novo paradigma de sustentabilidade. Revista Gestão e Desenvolvimento em ContextoGEDECON Edição Especial - Porangatu, v.2, n.1, p.146-157, jan. /jul. 2013.

OLIVEIRA, Sulayre Mengotti de. Aproveitamento da água da chuva e reúso de água em residências unifamiliares: estudo de caso em Palhoça - SC, junho de 2005.

OPAS (2001) Água e Saúde.Relatório.www.opas.org.br/sistema/fotos/agua.pdf. $<$ Acessado em abril de 2017>

PALHOÇA, Prefeitura Municipal de Palhoça. Disponível em: http://palhoça.sc.gov.br, acessado em novembro de 2004.

PROSAB, Uso Racional da Água em Edificações-Ricardo Franci Gonçalves (Coord.). Rio de Janeiro : ABES, 2006

SETTI, A. A. A Necessidade do Uso Sustentável dos Recursos Hídricos. IBAMA, Brasília, 1994.

SICKERMANN, Jack M. Gerenciamento Sustentável das Águas de Chuva: imprescindível para o futuro das grandes cidades do Brasil. Piauí, 2005. 9p.

SILVEIRA, Bruna Quick da. REUSO DA ÁGUA PLUVIAL EM EDIFICAÇÕES RESIDENCIAIS, janeiro de 2008.

TOMAZ, P. Economia de água para empresas e residências: um estudo atualizado sobre o uso racional da água. $2^{\circ}$ Edição. São Paulo: Navegar Editora, 2005. 
VESENTINI, J. W. Brasil, sociedade e espaço. 7. ed. São Paulo: Ática, 1999.

ZAMPIERON, Sônia Lúcia Modesto; VIEIRA, João Luiz de Abreu. "Poluição da Água". Disponível em: <http://educar.sc.usp.br/biologia/textos/m_a_txt5.html>

Enviado: Noviembre, 2019.

Aprobado: Enero de 2020. 